Torres Soto, A. \& Vallejo Ruiz, M. (2018). ¿Contribuye la universidad al desarrollo de aprendizajes de calidad? Estudio descriptivo con estudiantes de la Universidad de Murcia. Revista Electrónica Interuniversitaria de Formación del Profesorado, 21(1), 129-142.

DOI: http://dx.doi.org/10.6018/reifop.21.1.295261

\title{
¿Contribuye la universidad al desarrollo de aprendizajes de calidad? Estudio descriptivo con estudiantes de la Universidad de Murcia
}

Ana Torres Soto, Mónica Vallejo Ruiz

Universidad de Murcia, España

\section{Resumen}

Este trabajo tiene como finalidad analizar la formación de un grupo estudiantes de Pedagogía valorando, específicamente, la profundidad del aprendizaje desarrollado a lo largo de su formación universitaria. Este estudio se ha abordado desde un diseño de estudio de caso utilizando un método no experimental descriptivo, en el que se ha aplicado un cuestionario diseñado ad hoc que recoge el conocimiento del alumnado a partir de la resolución de varias situaciones problemáticas propias de su ámbito profesional. Al tratarse de un caso concreto, se ha aplicado a los 47 estudiantes de Pedagogía de la Universidad de Murcia que se encontraban en el último curso. Los resultados muestran que un mayor número de estudiantes ha desarrollado un aprendizaje superficial, evidenciándose cotas de comprensión bajas y la resolución simple de problemas; pudiendo dilucidar, por tanto, que tienen dificultades para argumentar sus respuestas y para resolver problemas relevantes de su ámbito profesional.

\section{Palabras clave}

Enseñanza universitaria; aprendizaje significativo; calidad de la educación; taxonomía; evaluación del aprendizaje.

\section{Is the university contributing to the development of quality learning? A study with students of Pedagogy}




\section{Abstract}

This paper aims to analyze the competential formation of a group of Pedagogy students, valuing, specifically, the depth of learning developed throughout their university education. This study has been approached from a case study design using a non-experimental descriptive method, in which a questionnaire designed ad hoc has been applied that collects the students' knowledge from the resolution of several problematic situations of their professional scope. As it is a concrete case, it has been applied to the 47 students of Pedagogy of the University of Murcia who were in the last course. The results show that a greater number of students has developed a superficial learning, evidencing low levels of understanding and simple problem solving; Being able to elucidate, therefore, that they have difficulties to argue their answers and to solve relevant problems of their professional scope.

\section{Key words}

University teaching, meaningful learning, quality education, taxonomy, learning assessment.

\section{Introducción}

El proceso de incorporación al Espacio Europeo de Educación Superior (EEES) abre un nuevo periodo de reflexión y revisión del quehacer docente, favoreciendo, asimismo, una oportunidad para experimentar nuevas formas de enseñar, aprender y evaluar en la universidad. Las numerosas experiencias piloto de adaptación al Grado, que fueron iniciadas en diversas universidades españolas, y los posteriores estudios de Grado se impulsaban con el propósito, entre otros, de favorecer la calidad del aprendizaje de los estudiantes. Esto implica promover el aprendizaje autónomo, sentar las bases del aprendizaje permanente y favorecer aprendizajes significativos y profundos que permitan la realización de actividades relevantes y significativas en contextos de actividad diversos y funcionales (Mauri, Colomina y Rochera, 2006; Pérez Gómez, Soto Gómez, Sola Fernández y Serván Núñez, 2009). Así, las universidades comienzan a introducir cambios metodológicos basados en la incorporación de metodologías activas, tratando de establecer una conexión entre teoría y práctica y el paso de una formación meramente disciplinar a otra centrada en lo académico y lo profesional.

La asunción de cambios de tal envergadura nos lleva a preguntarnos si realmente se están provocando aprendizajes funcionales y relevantes en los estudiantes, dada la importancia que se otorga al desarrollo de competencias y a la formación para la empleabilidad en el contexto actual. Además, algunos autores (Biggs, 2006; Bowden y Marton, 2012; Prieto, Blanco, Morales y Torre, 2008; Rué, 2007) consideran que, en este escenario de cambio hacia una formación más competencial, se hace oportuno y necesario revisar los resultados de aprendizaje de los estudiantes para replantear los modos de enseñar. En efecto, si se pretende emprender la mejora de la calidad de la enseñanza se debe conocer los resultados de las experiencias de los estudiantes y las consecuencias de su aprendizaje. En este sentido, este estudio pretende conocer los resultados de aprendizaje de los estudiantes de Pedagogía de la Universidad de Murcia en relación a su conocimiento funcional -referido a la capacidad del alumno en la ejecución y resolución de problemas relacionados con su profesión-, para reflexionar, consiguientemente, sobre la calidad de la enseñanza recibida. 


\section{Hacia un aprendizaje profundo en contextos de cambio e incertidumbre}

Hace algunos años numerosas universidades asumieron el compromiso de reconsiderar sus metodologías docentes y orientarlas hacia el protagonismo de los estudiantes y hacia el desarrollo de competencias profesionales y transversales encaminadas no sólo a la empleabilidad, sino también a la formación integral de los estudiantes (Silva Laya, 2016). El reto que aceptan dichas universidades implica aportar un valor añadido (Martínez y Viader, 2008) a la formación de sus egresados, que les confiera un carácter flexible e integral ante la incertidumbre del mundo laboral. Se plantea, en este aspecto, la necesidad de formar a los estudiantes para pensar, decidir y actuar en la vida real (Vallejo y Molina, 2014). Estos planteamientos son el eje central de un debate educativo a nivel internacional -y mundialque impulsan la formación a partir de competencias para la formación integral del los estudiantes.

Sin embargo, alcanzar este tipo de formación no es tarea sencilla. Numerosos autores (Gargallo, Garfella, Sahuquillo, Verde, Jiménez, 2015; Huber, 2008) afirman que las implicaciones de la enseñanza y el aprendizaje en las universidades actuales requieren de nuevos papeles y responsabilidades para docentes y discentes. Según Huber (2008), urgen cambios en los procesos de enseñanza y aprendizaje encaminados hacia la alta capacitación de los estudiantes. Fundamentándonos en una amplia revisión bibliográfica (Marton, Dall'Alba y Beaty, 1993; Hopkins, 2000; Darling-Hammond, 2001; Hativa, 2001; Ramsden, 2003; Domingo, 2003; Biggs, 2006; Pozo, 2006; Bain, 2007, 2014; Huber, 2008; Rué, 2009; Biggs y Tang, 2011; Bowden y Marton, 2012), podríamos situar esa capacitación en el desarrollo de los niveles más elevados de aprendizaje, y ello supone, a grandes rasgos, dotar de significado el conocimiento que se adquiere y ser capaz de aplicarlo a situaciones diversas.

Estas exigencias requieren de esfuerzos (por parte de la universidad y de los agentes implicados en los procesos de enseñanza y aprendizaje) encaminados a cambiar las prácticas de enseñanza y, con ello, aquellos factores que favorecen la dimensión más conceptual y teórica del conocimiento. Todo ello pasa por encaminar el aprendizaje del alumnado, por un lado, hacia el desarrollo de concepciones complejas y enfoques profundos de aprendizaje, y por otro, hacia la enseñanza de técnicas y estrategias adecuadas de aprendizaje (Salgado-Horta y Maz-Machado, 2013). En este mismo sentido, Pérez Gómez et al. (2009) manifiestan que la clave del aprendizaje de calidad se encuentra en provocar en los estudiantes aprendizajes relevantes y eficaces, en cuanto que sirvan para comprender y afrontar problemas básicos y ampliar los horizontes del conocimiento. Puntualizan que además de enseñar el conocimiento de hechos o teorías, sería crucial también enseñar su operatividad y utilidad, $y$, sobre todo, el sentido de dichos conocimientos para el desarrollo del propio proyecto personal y profesional. De hecho, es asumida la idea de que para que los estudiantes aprendan profundamente es indispensable dotar de sentido aquello que van a aprender (Ramsden, 2003).

En trabajos de diversos autores (Biggs y Collis, 1982; Rué, 2009; Biggs y Tang, 2011; Ritchhart, Church y Morrison, 2014) se afirma que el aprendizaje de los estudiantes debe ser profundo y significativo. De esta manera, sitúan el aprendizaje entre dos polos; un extremo relacionado con el aprendizaje superficial, fruto de un conocimiento memorístico, cuantitativo y reproductivo; y el otro extremo relacionado con el aprendizaje profundo, derivado de un conocimiento constructivo, reflexivo y cualitativo. En relación a este aspecto, existen diversas taxonomías que han tratado de ejemplificar los niveles de 
complejidad y calidad de los aprendizajes de los estudiantes. Biggs y Collis (1982) elaboraron una taxonomía denominada SOLO (acrónimo de 'Estructura de los resultados de aprendizaje observados'), que viene a caracterizar cinco niveles de complejidad estructural en el aprendizaje de los estudiantes, que avanza desde los niveles más simples y superficiales del conocimiento (niveles preestructural, uniestructural y multiestructural) hasta los niveles más profundos (niveles relacional y de abstracción extendida). Por ofrecer una pincelada de lo que estos autores determinaron, en la Figura 1 se da cuenta de la estrecha relación de los niveles propuestos en dicha taxonomía, los enfoques de aprendizaje adoptados por los estudiantes y la comprensión adquirida según los procesos cognitivos que haya puesto en marcha el alumno para aprender.

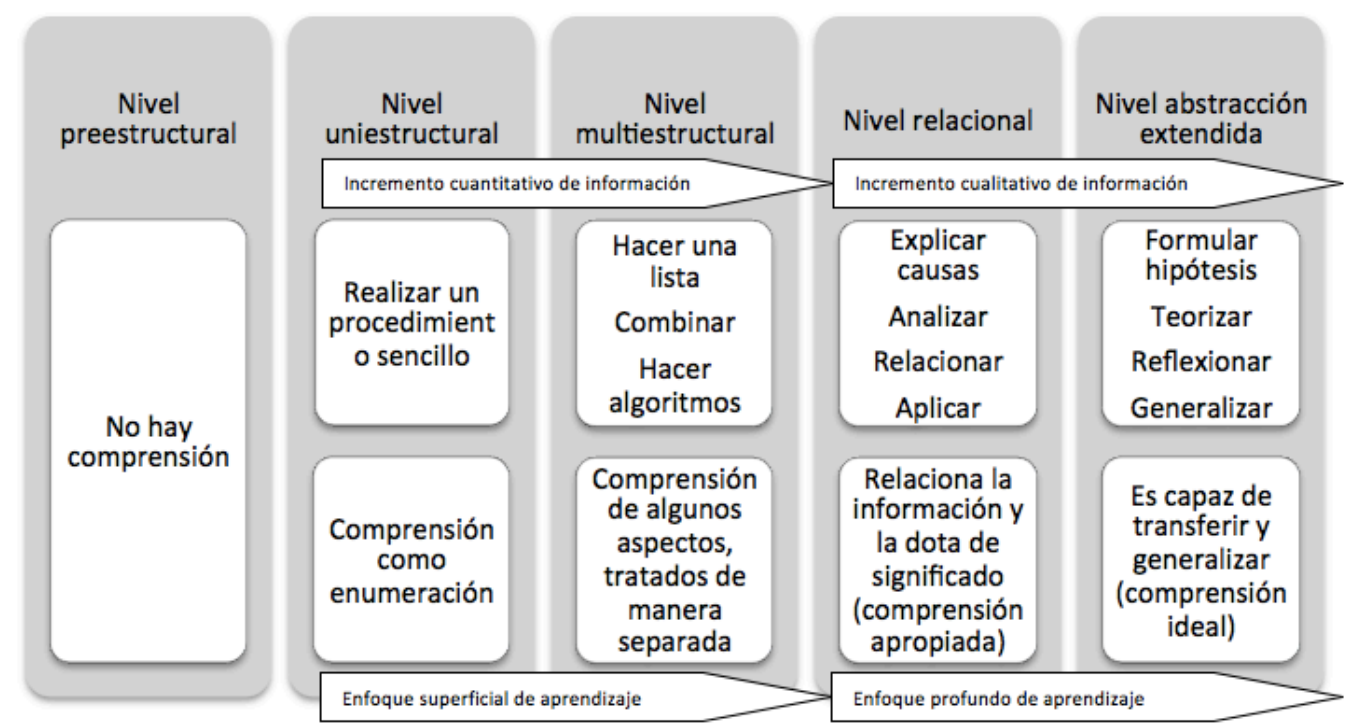

Figura 1. Niveles de la taxonomía SOLO y su relación con la comprensión

Resulta evidente que la transferencia del conocimiento y su aplicación a diversas situaciones profesionales, requiere de la puesta en marcha de los niveles más elevados de la taxonomía. Respecto a esta idea, son muchos los autores (Bain, 2007; Biggs, 2006; Marton y Säljo, 1976a, 1976b; Entwistle, 1986; Biggs, 1987) que se han posicionado manifestando la necesidad de desarrollar concepciones de aprendizaje complejas y enfoques de aprendizaje profundos, para mejorar la calidad del aprendizaje de los estudiantes.

De acuerdo con las ideas expuestas, este artículo trata de motivar la reflexión en torno a las siguientes cuestiones: ¿Qué tipo de aprendizaje se está desarrollando en la Universidad? ¿Están construyendo los estudiantes un aprendizaje profundo? ¿Se está capacitando a los estudiantes para la resolución de problemáticas profesionales? ¿Razonan sobre las implicaciones y aplicaciones de lo que aprenden? Estas cuestiones nos ayudan a situar el punto de partida de nuestro trabajo cuyo objetivo se dirige al estudio de los resultados de aprendizaje de los estudiantes de Pedagogía de la Universidad de Murcia, que se formaron en el periodo de adaptación a los nuevos grados. Se ha de considerar que dichos estudiantes fueron partícipes de Proyectos de Innovación Educativa que pretendían la adaptación de los diversos cursos a algunos planteamientos de la Convergencia Europea, entre los que se encuentran, principalmente, metodologías orientadas al desarrollo de competencias y la utilización de una gran variedad de procedimientos de evaluación coherentes con la metodología empleada y con las competencias a valorar. 


\section{Metodología}

\section{Diseño de la investigación}

La estrategia utilizada en el diseño de la investigación ha sido el estudio de caso, teniendo éste una finalidad evaluativa (Pérez Serrano, 1994). Específicamente, se trata de un diseño de investigación transeccional descriptivo ya que el objetivo de este estudio fue conocer una realidad determinada (aprendizaje funcional de los estudiantes) en un momento determinado. Este estudio se encuentra en contacto directo con el objeto de estudio y en su propio contexto natural (el aula), con la intención de no alterar el sentido o significados que allí confieren los estudiantes a sus experiencias de aprendizaje (Merriam y Tisdell, 2016). En este aspecto, se ha buscado, precisamente, especificar los hallazgos más que generalizarlos, dada la aplicación localista, el contexto y el significado de las interpretaciones subjetivas de los participantes.

La investigación se ha diseñado a partir de los resultados obtenidos en un estudio previo que analizaba el conocimiento declarativo (conceptual) de la muestra de estudiantes que aquí se ha utilizado (véase Torres, 2015). Este estudio previo, realizado también a través de un diseño transeccional descriptivo, supuso un primer contacto con los estudiantes a través del cual se valoró el conocimiento declarativo acerca de diez contenidos representativos del Plan de Estudios.

\section{Contexto y participantes}

La selección de la muestra del estudio se llevó a cabo mediante un muestreo no probabilístico basado en la intención (Ruiz Olabuénaga, 2012), ya que se pretendía recoger información en profundidad de un grupo con unas características deseadas. Concretamente, la muestra estuvo conformada por 47 estudiantes ( 43 chicas y 4 chicos) de Pedagogía de la Universidad de Murcia que se encontraban finalizando el último curso académico del título. Estos estudiantes reunían una característica singular: haber comenzado la Titulación de Pedagogía en el curso académico 2007/2008. El motivo de plantear dicho criterio obedecía a nuestro interés por investigar el aprendizaje de estudiantes que tuvieran una formación similar (en cuanto a materias, docentes que las impartieran y duración de estudios).

Se eligió la titulación de Pedagogía porque consideramos que la formación del pedagogo debe ocupar, por los diversos motivos que se exponen seguidamente, una atención especial. Este presenta un perfil competencial con una extensa variabilidad de funciones que, a juzgar por el desconocimiento y escaso reconocimiento de estos estudios, han abocado en un intrusismo profesional (Álvarez y Romero, 2007). Estas características nos llevaron al propósito de valorar la formación de los pedagogos, visibilizando y caracterizando algunas de las actividades que estos profesionales pueden desempeñar.

\section{Instrumento}

Partiendo de nuestros objetivos de investigación y de la muestra participante era preciso diseñar un instrumento ad hoc que nos permitiera valorar el conocimiento de los estudiantes. Así, se diseñó un cuestionario semiestructurado comprendido de tres casos prácticos (afines a las funciones que debe desempeñar un egresado en Pedagogía en su ámbito profesional) que nos permitieran acceder al conocimiento funcional de los estudiantes, de manera que sus respuestas fuesen dirigidas a enunciar contenidos, explicarlos, argumentarlos y aplicarlos. Entre estas funciones se encuentran las siguientes: analizar situaciones educativas (primer caso práctico), realizar seguimientos y evaluaciones (segundo caso práctico), y diseñar programas, acciones y proyectos (tercer caso práctico). 
Seguidamente se presentan los tres supuestos prácticos planteados y los temas sobre los que han versado:

\section{Supuesto 1. Detección de un alumno con altas capacidades.}

En este supuesto se planteó al estudiante que, imaginando su labor como pedagogo en un Equipo de Orientación Educativa y Psicopedagógica (EOEP), respondiera a diversas cuestiones para la detección de un alumno de tercer curso de Primaria con supuestas altas capacidades. Concretamente, las cuestiones planteadas para dirigir las respuestas de los estudiantes han sido las siguientes: ¿Cómo determinarías si se trata de un alumno con altas habilidades? ¿Utilizarías alguna prueba o test específico? ¿Cuál o cuáles serían? ¿Dónde los localizarías? ¿Qué información incluirías en el Informe psicopedagógico? ¿Sería necesario hacer un dictamen de escolarización? ¿En qué caso? ¿Solicitarías ayuda a algún tipo de agente o servicio? ¿Cuál? Imagina que, tras la evaluación psicopedagógica, determinas que se trata de un alumno superdotado. ¿Qué medidas contemplarías para dar respuesta educativa a las necesidades educativas del alumno?

Supuesto 2. Importancia e implicaciones de la evaluación en educación.

El segundo supuesto que se ha planteado incide sobre el conocimiento de los alumnos acerca de la evaluación. En este caso se pretendía que el alumnado pusiera de manifiesto su capacidad de asesoramiento y orientación a profesores sobre los tipos distintos tipos de evaluación, los instrumentos de evaluación y las implicaciones de su uso en la función docente. Asimismo, se intentó que el alumnado se posicionase ante los distintos tipos de evaluación considerando no solo sus conocimientos sino también su experiencia de aprendizaje. Las cuestiones planteadas en este supuesto fueron las siguientes: ¿Qué entiendes tú por evaluación de competencias? ¿Qué tipos de evaluación conoces? De los distintos tipos de evaluación que has definido, ¿cuál o cuáles debería emplear el profesorado y por qué? ¿Qué instrumentos les plantearías que utilizaran y en qué momento deberían utilizarlos? ¿Qué repercusión crees que tendría este tipo de evaluación en su práctica como docente?

Supuesto 3. Formación continua del profesorado.

El tercer y último supuesto recoge la necesidad de la formación continua del profesorado como uno de los planteamientos decisivos en el aprendizaje de los estudiantes. Concretamente, en este supuesto los estudiantes debían imaginar cómo llevar a cabo un programa de formación continua con el profesorado de secundaria y, para ello, se le planteó unas cuestiones imprescindibles para conocer su capacidad de análisis de situaciones así como para plantear acciones y medidas para la formación continua de profesores. Las preguntas que se plantearon en relación a esta temática fueron las siguientes: ¿En base a qué argumentos defenderías ante un grupo de profesores la importancia de la formación continua? Indudablemente, antes de implementar cualquier acción formativa has de considerar una serie de cuestiones previas (necesidades) que te permita diseñar el programa de formación de manera contextualizada. ¿Qué cuestiones previas tendrías en cuenta a la hora de diseñar un programa de formación? En muchas ocasiones, los aprendizajes que se pueden desarrollar en un curso o taller de formación del profesorado pueden darse con distintos niveles de intensidad: sensibilización, comprensión, desarrollo de habilidades, uso o reconstrucción/transferencia. ¿Cómo plantearías el curso si tu intención es que los docentes transfieran aquello que han aprendido a su práctica educativa? ¿Qué metodología de investigación planearías? Imagina que se trata de centro en el que, en los últimos años, se ha creado una cultura escolar tendente al individualismo por parte de los docentes. ¿Cómo abordarías un cambio en el que los docentes trabajaran 
de forma colaborativa? ¿Utilizarías algún recurso tecnológico? ¿Por qué? Explícalo brevemente.

El instrumento fue validado a nivel de contenido a través de una evaluación inter-jueces, como técnica analítica de triangulación, por expertos en campos de la Planificación de la enseñanza y Métodos de Investigación. Esa validez también era amparada por la validez de uso, es decir, por su utilización en diferentes estudios con grandes similitudes (García Hernández, 2009; Navarro Medina, 2012; Torres, Vallejo y Martínez Valcárcel, 2012). Respecto a la consistencia interna del instrumento, el Coeficiente Alfa de Cronbach arrojó un coeficiente de $\mathbf{0 . 8 1 4}$, lo cual indica, de acuerdo con Bisquerra (2012), una fiabilidad alta.

\section{Análisis de los datos}

Previo al análisis de los datos se elaboró una matriz que nos permitiera afrontar el análisis de la calidad de las respuestas de los alumnos, en la que se detallaron cinco niveles de progresión (1 menor y 5 mayor) en la calidad de las respuestas y tres categorías de análisis que permitieran dar respuesta a nuestro objetivo de investigación. Estas categorías se identifican con cada uno de los tres casos prácticos planteados, presentados en el subapartado precedente. Por su parte, el análisis de las tres categorías estudiadas se fundamentó en la taxonomía SOLO de Biggs y Collis (1982), posteriormente contemplada en Biggs (2006) y Biggs y Tang (2011). Estos niveles fueron adaptados y contextualizados de acuerdo con la temática de cada una de las categorías que se pretendía analizar. En la siguiente tabla figura un ejemplo de redefinición de los niveles de acuerdo con uno de los supuestos analizados (Tabla 1).

Tabla 1.

Matriz para el análisis de las respuestas de los alumnos

\section{Categoría Intervención Pedagógica alumno con altas capacidades}

Nivel $1 \quad$ No sabe cómo proceder ante la situación planteada. No es capaz de identificar ninguna prueba específica, ni contempla una respuesta pedagógica. (C1-N1)

Nivel 2 Conoce de qué manera tiene que proceder para determinar si se trata de un alumno con altas habilidades e incluso indica el nombre de alguna prueba específica. Sin embargo, no es capaz de discriminar entre un informe y un dictamen de escolarización. Además, las medidas que contempla para su respuesta educativa son confusas y poco definidas. El contenido de la respuesta es muy superficial y no entra en detalles. Solo da respuesta a algunas de las cuestiones planteadas. (C1-N2)

Nivel 3 Es capaz de dar respuesta a todas las cuestiones planteadas. Conoce alguna prueba específica, enuncia qué información incluiría en el informe psicopedagógico, pero tiene problemas para determinar cuándo tiene que realizar un dictamen de escolarización. Es capaz de enumerar algunas medidas para dar respuesta a las necesidades del alumno, sin embargo, no es capaz de establecer relaciones, explicar causas, etc. Recuerda conceptos relacionados con la respuesta a la diversidad educativa (adaptaciones curriculares significativas, no significativas, currículum individualizado, etc.), aunque no se muestran evidencias de comprensión. (C1-N3)

Nivel $4 \quad$ Además de dar respuesta a todas las cuestiones planteadas, es capaz de describir, de manera detallada, las pruebas específicas para diagnosticar al alumno. Sabe dónde localizar las pruebas y a quién solicitar ayuda si la necesitara. Sobre todo, cuando se le pregunta acerca de las medidas que contemplaría, incluye información muy detallada, fundamentado incluso sus respuestas. ( $\left.1-\mathrm{N}_{4}\right)$ 
Nivel 5 Es capaz de discutir, evaluar, interpretar y razonar la información proporcionada. Maneja conceptos elaborados e incluye razonamientos y conclusiones propias. Es capaz de argumentar diversas respuestas. Indica medidas ordinarias y extraordinarias, la importancia de sensibilizar al profesorado, del trabajo de las familias, etc. (C1-N5)

Se recurrió a la estadística descriptiva para el análisis de la información de carácter cuantitativa, la cual fue analizada a partir del programa de análisis estadístico SPSS/PC versión 19; y al análisis de contenido para analizar la información de carácter cualitativa, la cual fue analizada con el programa Atlas.ti.

\section{Resultados}

En este apartado se recoge un conjunto de consideraciones resultantes del trabajo de campo y del análisis de datos que arrojan información acerca de los niveles de conocimiento de los estudiantes de Pedagogía de la Universidad de Murcia en la resolución de tres casos prácticos que recogen diferentes situaciones auténticas del ámbito de desarrollo profesional del pedagogo. Las respuestas de los estudiantes se categorizaron de acuerdo con los niveles de la taxonomía SOLO (1-5). La estadística descriptiva de dichas categorías se recoge en la siguiente tabla.

Tabla 2.

Descriptivos sobre las categorías estudiadas

\begin{tabular}{lccc}
\hline & Media & Desviación típica & N del análisis \\
\hline \begin{tabular}{l} 
C1. Detección de un alumno con altas capacidades \\
\hline $\begin{array}{l}\text { C2. Importancia e implicaciones de la evaluación en } \\
\text { educación }\end{array}$
\end{tabular} & 3,15 &, 834 & 47 \\
\hline C3. Formación continua del profesorado & 3,36 &, 729 & 47 \\
\hline
\end{tabular}

A nivel global, se puede apreciar los valores medios obtenidos por los alumnos en las diferentes categorías. Dichos valores oscilan entre las puntuaciones 3,15 y 3,36, lo cual pone de manifiesto que el conocimiento de los estudiantes respecto a estas variables se sitúa en el nivel 3 o multiestructural. Una primera impresión de este resultado nos permite razonar que los estudiantes muestran un aprendizaje ligeramente profundo y comprensivo cuando tienen que poner en marcha su conocimiento funcional, esto es, resolver algunos problemas o casos prácticos vinculados con la detección de alumnos con altas capacidades, con la importancia e implicaciones de la evaluación en educación y con la formación continua del profesorado. Pero se puede observar ciertas limitaciones relacionadas con el análisis de la realidad, la interpretación y la reflexión argumentada de sus respuestas.

En la Figura 2 se presentan los porcentajes de respuesta de los estudiantes en cada nivel de la Taxonomía SOLO en cada una de las grandes categorías analizadas ( $C_{1}, C_{2}$ y C3).

De acuerdo con la columna Total, que engloba la totalidad de las respuestas en las tres categorías, el $49,6 \%$ de las respuestas de los estudiantes se sitúan en el nivel multiestructural. Al igual que se apreciaba en el análisis anterior, este dato evidencia que los estudiantes son capaces de responder correctamente a las situaciones prácticas 
planteadas, si bien la información expuesta en torno a los problemas planteados es demasiado genérica y poco elaborada. Casi un 35\% de las respuestas de los estudiantes (compuestas por el 29,8\% del nivel relacional y el 5\% del nivel de abstracción extendida), muestran un aprendizaje profundo sobre estas temáticas, basadas en la generación de juicios de valor, argumentaciones adecuadas y reflexiones justificadas. A continuación se presenta un análisis detallado de cada una de las categorías analizadas.

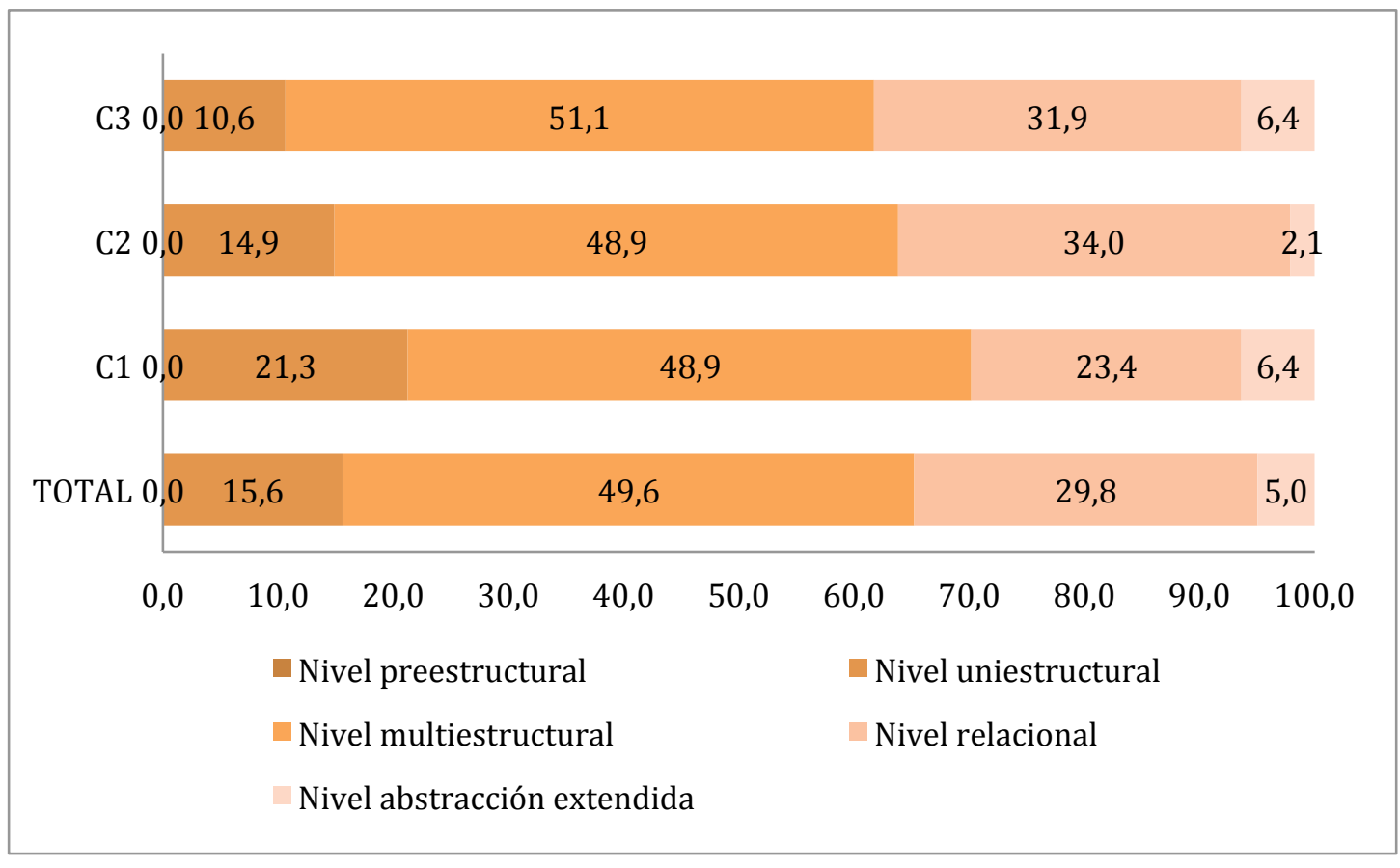

Figura 2. Porcentaje de respuestas obtenidas en cada categoría según niveles

\section{C1. Detección de un alumno con altas capacidades}

El análisis de las respuestas nos ha permitido evidenciar que todos los estudiantes conocen diversas pruebas que pueden ser utilizadas para diagnosticar a alumnos con altas capacidades. La diferencia entre unos estudiantes y otros se basa en la descripción que cada uno realiza sobre dichas pruebas. Asimismo, son capaces de enunciar qué cuestiones se incluyen en un informe psicopedagógico, aunque es destacable y curioso el desconocimiento casi generalizado de qué es y para qué se utiliza un dictamen de escolarización.

De otro lado, solo un tercio de los estudiantes (quienes se encuentran en los niveles relacional y de abstracción extendida) son capaces de razonar y argumentar qué respuesta educativa sería la adecuada para estos alumnos. Así y a modo de ejemplo, cuando se les pregunta qué medidas contemplarían para dar respuesta educativa a las necesidades educativas del alumno, los estudiantes cuyas respuestas se ubican en los niveles más bajos responden:

Ajuste en su proceso académico mediante agentes especializados (pedagogos, orientadores) y mediante metodologías más avanzadas y adecuadas a su nivel intelectual. [P23: Sujeto22.txt - 23:10 (135:160)]. 
Mientras que los estudiantes cuyas respuestas se hallan en los niveles más elevados contestan:

Se puede contemplar dos tipos de medidas: Ordinarias: Como adaptar los contenidos del currículum a su nivel (trabajo de investigación, más ejercicios o más elaborados), mandar ejercicios de temas más adelantados, trabajando la dinámica de grupos, el trabajo cooperativo, etc. Extraordinarias: como adelantar un nivel o ciclo, escolarizándolo en un centro específico. [P12: Sujeto11.txt - 12:12 (174:220)].

Se contempla que las respuestas ubicadas en los niveles más bajos son muy generales y no especifican respuestas educativas concretas. Estos estudiantes tienen dificultades para proporcionar una respuesta educativa exhaustiva a un estudiante con altas capacidades.

\section{C2. Importancia e implicaciones de la evaluación en educación}

El análisis de las respuestas en relación a esta categoría nos ha permitido comprobar que todos los estudiantes son capaces de enunciar cada uno de los tipos de evaluación que conocen y los instrumentos que utilizarían en cada una de dichas evaluaciones. La diferencia entre las respuestas más simples y más elaboradas las encontramos en que estas últimas no solo presentan la enumeración de tipos de evaluación (inicial, procesual, final, etc.) sino que también ofrecen la descripción y valoración de dichas evaluaciones. Concretamente, se observa que el $48,9 \%$ de los estudiantes (nivel multiestructural) se limita a la enumeración de las mismas y a indicar en qué momento del proceso de enseñanzaaprendizaje lo utilizarían. Mientras el 34\% de los estudiantes (niveles relacional) también ofrecen la descripción y valoración de dichas evaluaciones. En el nivel de abstracción extendida (2,1\%), se aprecia que, además de responder adecuadamente a las cuestiones planteadas, han utilizado razonamientos coherentes y relacionado los contenidos, han presentado ejemplos e incluso la translación a otros temas relacionados con los que se puede vincular la evaluación.

Por otro lado, cuando se les solicita a los estudiantes que reflexionen y analicen críticamente las implicaciones y repercusiones que pueden tener los diversos tipos de evaluaciones en la función del docente, solo las respuestas categorizadas en los niveles más altos (relacional y de abstracción extendida), evidencian una comprensión profunda respecto al tema:

Las repercusiones que tendría este tipo de evaluación para su práctica docente serían todas las de carácter positivo: les ayudaría a comprender y valorar desde una perspectiva diacrónica la evolución del alumnado y de su ejercicio profesional; a la hora de enfrentarse a una entrevista con padres tendría una gran variedad de información extraída de múltiples fuentes para fundamentar sus decisiones; les facilitaría realizar las modificaciones pertinentes para dar respuesta acertada a las necesidades educativas del alumnado; potenciaría, en definitiva, su desarrollo profesional. [P27: Sujeto26.txt - 27:11 (320:369)].

\section{C3. Formación continua del profesorado}

El análisis de esta categoría nos ha permitido evidenciar que todos los estudiantes son capaces de enunciar la importancia de la formación continua, la metodología que utilizarían y cómo plantearían el trabajo cooperativo; sin embargo, se aprecian diferencias en cuanto a la cantidad de información utilizada, adecuación y complejidad de la misma en las diversas respuestas. Un 51,1\% de las respuestas se ubican en el tercer nivel (multiestructural), caracterizado por un alumnado capaz de responder adecuadamente al caso práctico pero mediante un discurso excesivamente descriptivo, poco elaborado y carente de análisis y crítica. Asimismo, no se aprecia coherencia entre las ideas, ni se argumentan las razones de las decisiones que se toman. Por ejemplo, cuando tienen que argumentar qué harían para 
abordar un cambio en el que los docentes trabajaran de forma colaborativa y qué recursos tecnológicos utilizarían, los estudiantes de este nivel se limitan a indicar que utilizarían el trabajo colaborativo pero no detallan cómo trabajar colaborativamente (respuesta tautológica). Se detecta que conocen diversas herramientas que se pueden utilizar para favorecer el trabajo colaborativo de los profesores pero, dadas las respuestas, parece ser que no conocen exhaustivamente su función y no son capaces de argumentan las razones por las cuales utilizarlas:

Para abordar el individualismo del profesorado sería recomendable la utilización de técnicas de trabajo colaborativo como pueden ser la separación por grupo de trabajo pequeños en los que los miembros tengan distintos niveles. Esos grupos de trabajo tendrían que realizar una Webquest sobre innovaciones en la práctica docente. También pueden realizar blog de la asignatura comentando los aprendizajes dados, etc. [P31: Sujeto30.txt - 31:13 (255:282)].

En el nivel relacional (31,9\%) y el nivel de abstracción extendida $(6,4 \%)$ se aprecian respuestas más elaboradas, argumentadas y detalladas. Por ejemplo, en el último nivel los estudiantes son capaces de identificar la investigación-acción como una metodología que favorece la transferencia:

Plantearía la investigación en acción con el profesorado para que desde la práctica transfieran a lo aprendido y mayor saber contextualizado, así como favorecer la cultura de aprendizaje colaborativo entre los docentes que posteriormente puedan favorecer la adopción de esta cultura de aprendizaje y de formación del profesorado en los propios centros escolares [P32: Sujeto31.txt - 32:12 (307:346)].

Mientras que la mayoría de los estudiantes alude a la utilización de una metodología participativa y activa, pero no entran en la denominación y caracterización de metodologías concretas.

\section{Discusión y conclusiones}

Los resultados obtenidos en esta investigación ponen de manifiesto la necesidad de la comunidad universitaria de seguir trabajando para conseguir que los estudiantes alcancen aprendizajes de calidad. Los resultados hallados permiten concluir que estos estudiantes manifiestan un perfil de aprendizaje más próximo al superficial cuando tienen responder a diversas cuestiones relacionadas con contenidos propios de su formación académica. Este hallazgo apunta a que los estudiantes, en general, son capaces de proporcionar cuantiosa información cognitiva sobre un tema concreto, pero presentan ciertas dificultades para movilizar los conocimientos teóricos, estructurar la información de manera coherente, para razonar sobre ella y relacionarla con otros temas o para emitir juicios de valor. Es decir, estos estudiantes tienen ciertas limitaciones para responder a cuestiones que requieren de la implementación y puesta en marcha de competencias relacionadas con análisis crítico de la información y reflexión sobre la misma.

Como se ha indicado en el apartado teórico, el aprendizaje competencial exige poner en marcha los niveles más elevados de aprendizaje (niveles relacional y de abstracción extendida), ya que éstos se relacionan con habilidades más complejas como la contextualización de la información, la posibilidad de relacionarla con otros contenidos, de emitir valoraciones al respecto, de aplicarla a diversas situaciones; y en cuanto al desarrollo de estas habilidades, se aprecian limitaciones y lagunas importantes. Como apuntan los rasgos descriptivos de la taxonomía y su relación con los niveles de compresión de los estudiantes (detallado en la Figura 1), se ha podido corroborar en las respuestas de los estudiantes una comprensión débil, relacionada con una concepción reproductiva del aprendizaje. Por tanto, la actuación de los profesores, en este caso, se habría de orientar 
hacia la promoción de un aprendizaje profundo, que implique no solo aprender los pasos para resolver un problema, sino también saber transferirlos a otros contextos y situaciones problemáticas. En definitiva, el desarrollo de competencias de alto nivel está relacionado con el aprendizaje significativo y profundo, por lo que se ha de insistir y exigir no solo la adquisición de saberes teóricos y prácticos, sino provocar desafíos que impliquen la movilización de los distintos saberes, ajustar el nexo de unión entre los contenidos teóricos y prácticos y estimular a los estudiantes para que expongan sus puntos de vista.

Somos conscientes -por los diversos informes emitidos por la Universidad de Murcia- que se han realizado innovaciones metodológicas durante los periodos de adaptación al grado, sin embargo, se observa que el aprendizaje reproductivo y memorístico sigue siendo la realidad en la formación de los estudiantes. Estas conclusiones también nos permiten inferir que la mayoría de los estudiantes participantes en este estudio han podido adoptar un enfoque superficial al abordar las tareas de aprendizaje y que sus concepciones de aprendizaje se relacionan con la asunción de respuestas preestablecidas. Sin embargo, se trata de una hipótesis que podría guiar hacia la continuación y el avance de este estudio en torno a los enfoques y las concepciones de aprendizaje.

Por otro lado, si se contrastan estos resultados con las tasas de rendimiento y de éxito de la Titulación de Pedagogía del curso 2011/2012, los cuales alcanzan un porcentaje del 75,41\% y del $85,35 \%$, respectivamente (Unidad para la Calidad, 2015), podemos concluir que los estudiantes de Pedagogía -que de acuerdo con el estudio que hemos presentado muestran un nivel de conocimiento eminentemente superficial-, han superado sobradamente los requerimientos exigidos por esta titulación y por la Universidad. Esta apreciación invita a reflexionar sobre el tipo de aprendizajes que se están propiciando y las estrategias de enseñanza que se utilizan para favorecer estos niveles de conocimiento.

Los resultados muestran que la universidad está formando a futuros profesionales de Pedagogía para que identifiquen, enuncien, enumeren, cuando su cometido debería orientarse a la reflexión, generalización, transferencia, aplicación, etc. Es por ello que los procesos de enseñanza habrían de orientarse hacia la promoción de los alumnos hacia un nivel relacional y de abstracción extendida, de manera que éstos pasen del conocimiento superficial de la materia a profundizar en su comprensión. Se ha de considerar que la institución universitaria reclama una formación 'competencial', con el objetivo de que los estudiantes sean capaces de enfrentarse a las diversas situaciones que se le puedan presentar profesionalmente.

Sin duda, se están realizando grandes esfuerzos para adaptar la formación de los estudiantes a las exigencias de Europa. En este sentido, parece necesaria la difusión de prácticas de investigación de esta tipología, que adviertan a los docentes y a la comunidad universitaria, en general, de los resultados que se están alcanzando.

\section{Referencias}

Bain, K. (2007). Lo que hacen los mejores profesores universitarios. Barcelona: Publicacions de la Universitat de València.

Bain, K. (2014). Lo que hacen los mejores estudiantes de universidad. Valencia: Publicacions de la Universitat de València.

Biggs, J. (2006). Calidad en el aprendizaje universitario. Madrid: Narcea. 
Biggs, J. y Collis, K. (1982). Evaluating the Quality of Learning: the SOLO taxonomy. Nueva York: Academy Press.

Biggs, J. y Tang, C. (2011). Theaching for Quality Learning at University. What the student does $\left(4^{a}\right.$ Ed.). Nueva York, NY: Society for Research into Higher Education. Open University Press.

Bowden, J. y Marton, F. (2012). La Universidad. Un espacio para el aprendizaje. Madrid: Narcea.

Darling-Hammond, L. (2001). El derecho de aprender. Crear buenas escuelas para todos. Barcelona: Ariel.

Domingo, J. (2003). Dimensiones y escenarios del buen aprendizaje para todos. Revista Electrónica Iberoamericana sobre Calidad, Eficacia y Cambio en Educación, REICE, 1(2), 1-21.

Entwistle, N. J. (1986). Approaches to Learning in Higher Education: Effects of Motivation and Perceptions of the Learning Environment. Annual Meeting of the American Educational Research Association, San Francisco.

García Hernández, M. L. (2009). Los instrumentos de evaluación en la Licenciatura de Pedagogía de la UMU (2002-2007). Tesis de Licenciatura, Murcia, Universidad de Murcia (España).

Gargallo, B., Garfella, P. R., Sahuquillo, P. M., Verde, I., y Jiménez, M. A. (2015). Métodos centrados en el aprendizaje, estrategias y enfoques de aprendizaje en estudiantes universitarios. Revista de Educación, 370, 229-254.

Hativa, N. (2001).Teaching for effective learning in Higher Education. Amsterdam: Kluwer.

Hopkins, D. (2000). Powerful learning, powerful teaching and powerful schools. Journal of Educational Change, 1(2), 135-154.

Huber, G. L. (2008). Aprendizaje activo y metodologías educativas. Revista de Educación, número extraordinario, 59-81.

Martínez, M. y Viader, M. (2008). Reflexiones sobre aprendizaje y docencia en el actual contexto universitario. La promoción de equipos docentes. Revista de Educación, número extraordinario, 213-234.

Marton, F. y Säljö, R. (1976a). On qualitative differences in learning-I: Outcome and process. British Journal of Educational Psychology, 46, 4-11.

Marton, F. y Säljö, R. (1976b). On qualitative differences in learning-II: Outcome as a function of the learner's conception of the task. British Journal of Educational Psychology, 46, 115-127.

Marton, F., Dall'Alba, G., Beaty, E. (1993). Conceptions of learning. International Journal of Educational Research, 19(3), 277-300.

Mauri, T., Colomina, R., Rochera, M.J. (2006). Análisis de casos con TIC en la formación inicial del conocimiento profesional experto del profesorado. Revista Interuniversitaria de Formación del Profesorado, 20(3), 219-231.

Merriam, S. B., y Tisdell, E. J. (2016). Qualitative research: a guide to design and implementation (4th edition). San Francisco: Jossey-Bass. 
Navarro Medina, E. (2012). La enseñanza de la Historia de España y el desarrollo de las competencias ciudadanas. El conocimiento del alumnado al finalizar el Bachillerato. Tesis de doctorado, Murcia, Universidad de Murcia (España).

Pérez Gómez, A., Soto Gómez, E., Sola Fernández, M., Serván Núñez, M.J. (2009). Contextos y recursos para el aprendizaje relevante en la universidad. Madrid: Ediciones Akal.

Pérez Serrano, G. (1994). Investigación cualitativa. Retos, interrogantes y métodos. España: La Muralla.

Pozo, J. I. (2006). La nueva cultura del aprendizaje en la sociedad del conocimiento. En J.I. Pozo, N. Scheuer, M.P. Pérez, M. Mateos, E. Martín, y M. De La Cruz (Eds.), Nuevas formas de pensar la enseñanza y el aprendizaje (pp. 29-54). Barcelona: Graó.

Prieto, L., Blanco, A., Morales, P., Torre, J. C. (2008). La enseñanza universitaria centrada en el aprendizaje. Estrategias útiles para el profesorado. Barcelona: Octaedro.

Ramsden, P. (2003). Learning to Teach in Higher Education ( $2^{\mathrm{a}}$ Ed.). Londres: Routledge.

Ritchhart, R., Church, M., Morrison, K. (2014).Hacer visible el pensamiento. Cómo promover el compromiso, la comprensión y la autonomía de los estudiantes. Paidós: Buenos Aires.

Rué, J. (2007). Enseñar en la Universidad. El EEES como reto para la Educación Superior. Madrid: Narcea.

Rué, J. (2009). El Aprendizaje Autónomo en Educación Superior. Madrid: Narcea.

Salgado-Horta, D. y Maz-Machado, A. (2013). Toma de apuntes y aprendizaje en estudiantes de Educación Superior. Revista Complutense de Educación, 24(2), 341-358. doi: http://dx.doi.org/10.5209/rev_rced.2013.v24.n2.42083

Silva Laya, M. (2016). Competencias de estudiantes iberoamericanos al finalizar la educación secundaria alta. Perfiles Educativos, 38(152), 88-108.

Torres, A. (2015). El conocimiento de los estudiantes como punto de partida para la mejora de la enseñanza. Tesis de doctorado, Murcia, Universidad de Murcia (España).

Torres, A. Vallejo, M. y Martínez Valcárcel, N. (2012). El recuerdo del aprendizaje de los estudiantes como referente para la reflexión del docente: el caso de la Licenciatura de Pedagogía de la Universidad de Murcia (2005-2010). Revista de currículum y formación del profesorado, 16(2), 419-436.

Unidad para la Calidad (2015). Informe de los resultados académicos. Universidad de Murcia.

Vallejo, M. y Molina, J. (2014). La evaluación auténtica de los procesos educativos. Revista iberoamericana de Educación, 64, 11-25. 